\title{
Numerical modelling of concrete curing, regarding hydration and temperature phenomena
}

\author{
M. Cervera ${ }^{\text {a }}$, R. Faria ${ }^{\text {b,* }}$, J. Oliver ${ }^{\text {a }}$, T. Prato ${ }^{\text {a }}$ \\ a E.T.S. Ingenieros de Caminos, Canales y Puertos, Technical University of Catalonia, C/Gran Capitán s/n, \\ Edificio C1, 08034 Barcelona, Spain \\ ${ }^{\mathrm{b}}$ Faculdade de Engenharia, Departamento de Engenharia Civil (Estruturas), Universidade do Porto, Rua Dr. Roberto Frias s/n, \\ 4200-465 Porto, Portugal
}

Received 27 February 2001; accepted 4 May 2002

\begin{abstract}
A numerical model that accounts for the hydration and aging phenomena during the early ages of concrete curing is presented in a format suitable for a finite element implementation. Assuming the percolation of water through the hydrates already formed as the dominant mechanism of cement hydration, the model adopts an internal variable called hydration degree, whose evolution law is easily calibrated and allows an accurate prediction of the hydration heat production. Compressive strength evolution is related to the aging degree, a concept that accounts for the influences of the hydration and curing temperature on the final mechanical properties of concrete. The model capabilities are illustrated by means of a wide set of experimental tests involving ordinary and high performance concretes, and through the simulation of the concrete curing on a viaduct deck of the Öresund Link.
\end{abstract}

(c) 2002 Elsevier Science Ltd. All rights reserved.

Keywords: Concrete; Curing; Hydration; Temperature; Aging; Modelling

\section{Introduction}

A rising tendency to use concrete as a structural material at early ages, that is, before completion of the hydration process, is observed nowadays. This is evident for pre-casting as well as for pre-stressed concrete, where anticipation of the loading of concrete provides economical benefits concerned with the reusing of formworks, as well as abbreviating the construction phase. Another field were high cadence in production is registered concerns rolled compacted concrete dams, where construction technologies resemble the ones usually adopted for roads. The quality control inherent to these situations is a subject of major concern, since durability and functionality of the structures is greatly affected by

\footnotetext{
${ }^{*}$ Corresponding author. Tel.: +351-22-508-1950; fax: +35122-508-1835.

E-mail address: rfaria@fe.up.pt (R. Faria).
}

undesirable damages like cracking, at early ages primarily associated with thermal and shrinkage effects.

A domain of research to which the present paper is mostly concerned refers to the modelling of such phenomena in a format suitable for structural applications, namely by providing a mathematical representation of the observed temperature and volume changes that take place in concrete associated to the hydration of cement, as well as the increase of strength and stiffness of the hardening paste due to aging. The model to be presented herein accounts for many of the relevant features of concrete behaviour at early ages, but in order to make it appropriate for an implementation within the context of the finite element method, the kinetics of the chemical reaction will be viewed on a macroscopic level, that is, at the scale of the specimens tested on laboratory.

Basically such approach corresponds to visualise the enhancement of the mechanical properties of concrete as due to changes in the concentration of the many 
constituents existing at the microscale, rather than an actual change of the mechanical properties of such components. Within this framework the aging process is therefore linked to the formation of new hydrates, and consequently the essential phenomenon which dominates the hydration kinetics is the diffusion of the free water between the layers of the already formed hydrates [1-3], here reproduced via a normalised variable termed 'hydration degree'. Its evolution obviously depends on the current value of the hydration degree, but owing to the thermally activated nature of cement hydration a strong dependency on the temperature is also observed, a feature usually accomplished by assuming an Arrhenius type kinematics.

Additionally an aging model is proposed to describe the compressive strength enhancement during the hydration process. This is based on the novel concept of 'aging degree', with a rate evolution here linked both with the hydration degree and with the curing temperature. Like in models purely based on maturity concepts this accounts for the influence of the temperature on the compressive strength evolution, but additionally it allows to capture the effect of the curing temperature history on the final concrete strengths.

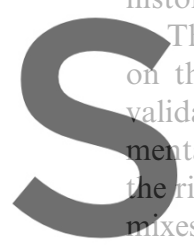

The model parameters inv

the basis of adiabatic o

alidation of the proposed $n$

tal tests concerning the

ising of the compressive under controlled cas
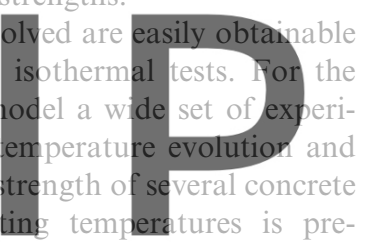

sented. Besides, it is demonstrated that the proposed

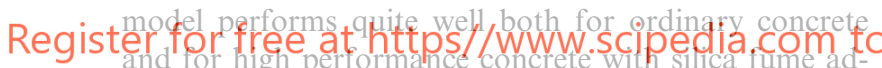
mixtures.

The article closes with a structural application, concerned with the simulation of the curing of a concrete deck of one of the viaducts of the Öresund Link (between Denmark and Sweden). Since during the concrete casting the selected viaduct was extensively monitored, comparison of the experimentally registered temperatures with the ones obtained via a numerical implementation of the proposed formulation will provide a fruitful discussion concerning the capabilities of the model here presented.

\section{Hydration of concrete}

\subsection{Thermo-chemical model}

The chemical processes associated to the hardening of concrete in the first days after casting are accompanied by significant temperature changes, since cement hydration is a highly exothermic and thermally activated reaction. Based on the theory of reactive porous media developed by Coussy in [1], as well as on its application to concrete under the macroscopic approach proposed by Ulm and Coussy in [2,3], the thermo-chemical model to be presented next is based on the macroscopic interpretation of the cement hydration as a chemical reaction in which the free water is a reactant phase, which combines with the unhydrated cement to form hydrates as a product phase.

Since the dominant mechanism in the kinetics of the reaction is due to the dispersion of water, if one defines $\chi$ as the number of moles of water combined per unit volume, this entity can be considered an internal variable of the system. For a more suitable handling and interpretation let us define this internal variable in the normalised format

$\xi=\frac{\chi}{\bar{\chi}_{\infty}}$

where $\bar{\chi}_{\infty}$ is the ideal final value of $\chi$ (attained in perfect conditions of the water/cement ratio w/c and other factors ensuring full hydration) and $\xi$ is what one could call the hydration degree, an indication of how much the hydration reaction has already advanced. In practical conditions the full hydration of the cement is not ob-

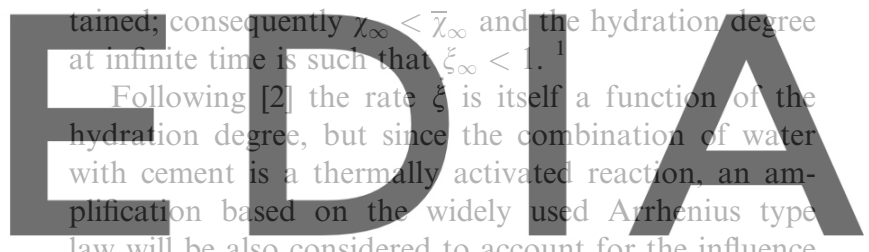

law will be also considered to account for the influence of the temperature [5]. A positive evolution of the form download the version without the watermark

$\dot{\xi}=\tilde{A}(\xi) \exp \left(-\frac{E_{\mathrm{a}}}{R T}\right) \geqslant 0$

is therefore proposed [2,3], where apart from the temperature $T$ the Arrhenius exponential term incorporates the constant $R$ for the ideal gases and the activation energy of the reaction $E_{\mathrm{a}}$. Eq. (2) encompasses the direct influence of the actual value of the hydration degree on term $\widetilde{A}$, a normalised affinity to be considered specific for each hydration reaction, and easily obtainable from an adiabatic calorimetric test, as it will be clarified later. In the definition of the normalised chemical affinity it is convenient to express it as

$\widetilde{A}=A \eta$

where $A$ is the chemical affinity (strictly speaking) and $\eta$ expresses the permeability directly linked with the microcirculation of the free water through the hydrates. By

\footnotetext{
${ }^{1}$ By relating $\xi_{\infty}$ with w/c in [4] it is suggested that the formula $\xi_{\infty}=1.031(\mathrm{w} / \mathrm{c}) /(0.194+\mathrm{w} / \mathrm{c})$ could be used for an estimation of the final hydration degree.
} 
assuming that the percolation of the water is retarded with the growth of the hydrates, $\eta$ would have to decrease with $\xi$, and consequently induced by [6] it is here postulated that

$\eta=\frac{1}{n_{0}} \exp \left(-\frac{\bar{n} \xi}{\xi_{\infty}}\right)$

where $n_{0}$ and $\bar{n}$ are material constants.

Now, by defining the chemical affinity with a format like [7]

$A=k\left(\frac{A_{0}}{k \xi_{\infty}}+\xi\right)\left(\xi_{\infty}-\xi\right)$

where $A_{0}$ is the initial affinity of the reaction (that is, when $\xi=0$ ) and $k$ is an additional parameter, handling

Eqs. (2)-(5) the final form for $\dot{\xi}$ reads

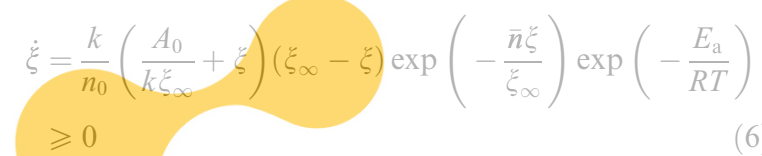

From the observation of this rate equation it becomes evident that the thermal activation expressed by the Arrmenius factor is of primary ginning of the reaction, but a

considerable attenuation is introduced b.

ability exponential term as well

lling the evolution as the hydration

Therefo
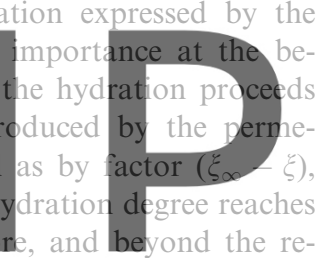

quired positiveness for $\xi$, the evolution expressed in Eq. Registerforipr the basigrefuigites for a realisticmodelling to

\subsection{Thermal field equation}

\subsubsection{Thermo-dynamic equilibrium}

According to the first and second principles of thermodynamics a domain undergoing thermal changes is ruled by a field equation of the form

$C \dot{T}=\dot{Q}+\dot{H}-\nabla \cdot Q_{\mathrm{f}}+\dot{D}$

where $C$ denotes the heat capacity per unit volume, $Q$ is the heat associated to an internal source, $H$ is the external heat source, $\boldsymbol{Q}_{\mathrm{f}}$ is the heat flux and $\dot{D}$ is the dissipation. Focusing on the simulation of the hydration problem it will be assumed that $C$ is constant, the dissipation is taken as negligible, the same occurring with the external heat source. Furthermore, the internal heat source $Q$ is provided by the hydration reaction itself, and according to many authors [6,8-10] hereafter it will be assumed that $\dot{Q}=Q_{\xi} \dot{\xi}$, with $Q_{\xi}$ being a material constant. Therefore Eq. (7) turns into

$C \dot{T}=Q_{\xi} \dot{\xi}+k_{\mathrm{T}} \nabla \cdot(\nabla T)$ where $\boldsymbol{Q}_{\mathrm{f}}=-k_{\mathrm{T}} \nabla T$ was assumed according to the Fourier's law ( $k_{\mathrm{T}}$ designates the thermal conductivity). Note that due to the dependency of $\dot{\xi}$ on $T$ the term due to the hydration heat $\dot{Q}$ acts as a nonlinear internal heat source, and accordingly an iterative procedure is usually required to solve Eq. (8) in space and time for general situations, as it will be discussed on the forthcoming section.

Besides the thermal field equilibrium expressed by Eq. (8), the Dirichlet and Newman boundary conditions have to be introduced. The first one concerns the temperature $\bar{T}$ prescribed along boundary $\Gamma_{T}$, whereas the second has to do with condition $\boldsymbol{Q}_{\mathrm{f}} \cdot \boldsymbol{n}=\bar{q}$, expressing the heat flux along the normal $\boldsymbol{n}$ to boundary $\Gamma_{q}$. In the applications to be presented in this paper $\bar{q}$ can be identified with the heat flux due to convection-radiation phenomena (if any), and accordingly one takes $\bar{q}=$ $h\left(T-T_{\text {ext }}\right)$, with $h$ being the convection-radiation coefficient and $T_{\text {ext }}$ denoting the external temperature.

\subsubsection{Finite element implementation \\ Following standard reasoning and assuming the usual interpolation strategy $T=N T^{\mathrm{e}}$, where $N$ de-

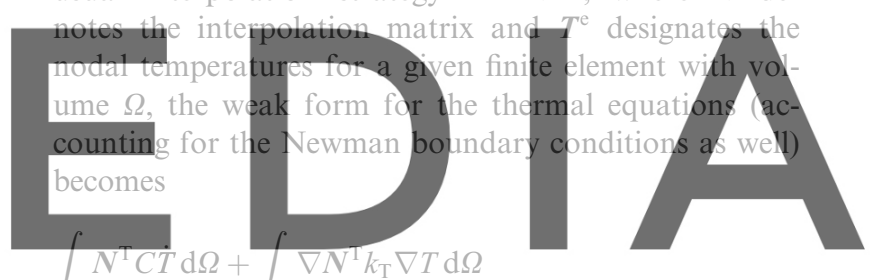 $\int_{\Omega} N^{\mathrm{T}} C \dot{T} \mathrm{~d} \Omega+\int_{\Omega} \nabla N^{\mathrm{T}} k_{\mathrm{T}} \nabla T \mathrm{~d} \Omega$ \\ downjoad the version without the watermark}

(if $\boldsymbol{N}$ trivially fulfils the Dirichlet conditions). Writing this equation for time $t_{n+1}$ and assuming a backwardEuler integration scheme of the form

$$
\begin{aligned}
& \dot{T}_{n+1}=\frac{\left(T_{n+1}-T_{n}\right)}{\Delta t} \\
& \dot{\xi}_{n+1}=\frac{\left(\xi_{n+1}-\xi_{n}\right)}{\Delta t}
\end{aligned}
$$

with $\Delta t$ being the time-step between two consecutive instants $t_{n}$ and $t_{n+1}$, Eq. (9) leads to the following equation, suitable for computational implementation:

$\frac{1}{\Delta t} \boldsymbol{C}^{\mathrm{e}}\left(\boldsymbol{T}_{n+1}^{\mathrm{e}}-\boldsymbol{T}_{n}^{\mathrm{e}}\right)+\boldsymbol{K}^{\mathrm{e}} \boldsymbol{T}_{n+1}^{\mathrm{e}}=\boldsymbol{F}_{T}^{\mathrm{e}}+\boldsymbol{F}_{\xi}^{\mathrm{e}}$

Following Eqs. (9)-(10b) the elemental matrices and 'force vectors' involved in this equation are computed according to

$$
\boldsymbol{C}^{\mathrm{e}}=\int_{\Omega} \boldsymbol{N}^{\mathrm{T}} C \boldsymbol{N} \mathrm{d} \Omega
$$




$$
\begin{aligned}
& \boldsymbol{K}^{\mathrm{e}}=\int_{\Omega} \nabla \boldsymbol{N}^{\mathrm{T}} k_{\mathrm{T}} \nabla \boldsymbol{N} \mathrm{d} \Omega+\int_{\Gamma_{q}} \boldsymbol{N}^{\mathrm{T}} h \boldsymbol{N} \mathrm{d} \Gamma_{q} \\
& \boldsymbol{F}_{T}^{\mathrm{e}}=\int_{\Gamma_{q}} \boldsymbol{N}^{\mathrm{T}} h T_{\mathrm{ext}} \mathrm{d} \Gamma_{q} \\
& \boldsymbol{F}_{\xi}^{\mathrm{e}}=\int_{\Omega} \boldsymbol{N}^{\mathrm{T}} Q_{\xi} \dot{\xi}_{n+1} \mathrm{~d} \Omega
\end{aligned}
$$

which through the using of standard assembling procedures will provide the global $\boldsymbol{C}$ and $\boldsymbol{K}$ matrices and the $\boldsymbol{F}_{T}$ and $\boldsymbol{F}_{\xi}$ vectors required for the finite element analysis of a structural problem, that is,

$$
\left(\frac{\boldsymbol{C}}{\Delta t}+\boldsymbol{K}\right) \boldsymbol{T}_{n+1}=\boldsymbol{F}_{T}+\boldsymbol{F}_{\xi}+\frac{\boldsymbol{C}}{\Delta t} \boldsymbol{T}_{n}
$$

Due to the dependency of the hydration rate on the temperature, expressed in Eq. (6), a two-level iterative procedure needs to be implemented to solve Eq. (13a): (i) at a structural level, due to the nonlinear dependency of $\boldsymbol{F}_{\xi}$ on $\boldsymbol{T}_{n+1}$; (ii) at a local level (that is, at each integration point), due to the nonlinear dependency of the

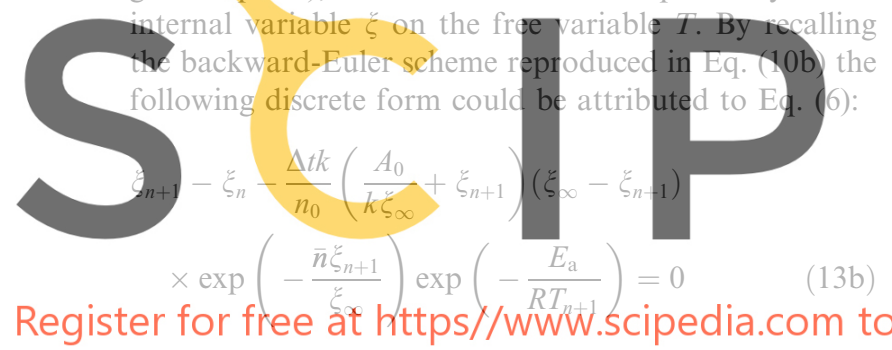

This equation is solved via the Newton-Raphson method, implemented at each integration point to extract the hydration degree $\xi_{n+1}$ associated to the local temperature $T_{n+1}$. This local temperature is obtained by performing a standard interpolation over the nodal temperatures $\boldsymbol{T}_{n+1}$, the latter provided by an 'outer' Newton-Raphson algorithm implemented to solve the nonlinear problem expressed at the structural level by Eq. (13a). After solving Eq. (13b) for $\xi_{n+1}$ the hydration rate $\dot{\xi}_{n+1}$ is computed from Eq. (10b), which according to Eq. (12d) provides a new trial vector for $\boldsymbol{F}_{\xi}$, and consequently an improved solution for $\boldsymbol{T}_{n+1}$ via Eq. (13a).

A remark is made to the fact that, since the rate evolution of $\xi$ is usually considerably greater than $\dot{T}$, the numerical integration of the hydration degree should be performed by using a time-step significantly lower than the one adopted for the structural problem [11]. Within the sub-incremental time-steps introduced to solve Eq. (13b) an interpolation is obviously required to define the sub-incremental values of the temperature at the integration point: the adoption of a linear interpolation between $T_{n}$ and $T_{n+1}$ is consistent with the time integra- tion scheme reproduced in Eq. (13b), and consequently it was assumed for the applications of the present paper.

\subsection{Experimental identification of the model parameters}

Under adiabatic conditions Eq. (8) becomes

$C \dot{T}=Q_{\xi} \dot{\xi}$

Denoting by $T_{0}$ the initial temperature this equation is easily solved to render

$T=T_{0}+Q_{\xi} \xi / C$

At the end of an adiabatic test $T=T_{\infty}$ and $\xi=\xi_{\infty}$, and consequently Eq. (15) can be used to evaluate the average value of constant $Q_{\xi}$, that is,

$Q_{\xi}=C\left(T_{\infty}-T_{0}\right) / \xi_{\infty}$

(16)

Furthermore, the normalised affinity $\mathcal{A}$, an essential entity of the hydration model, is also directly 'measurable' during the adiabatic test. By substituting Eq. (2) into Eq. (14) owing to Eq. (16) one obtains

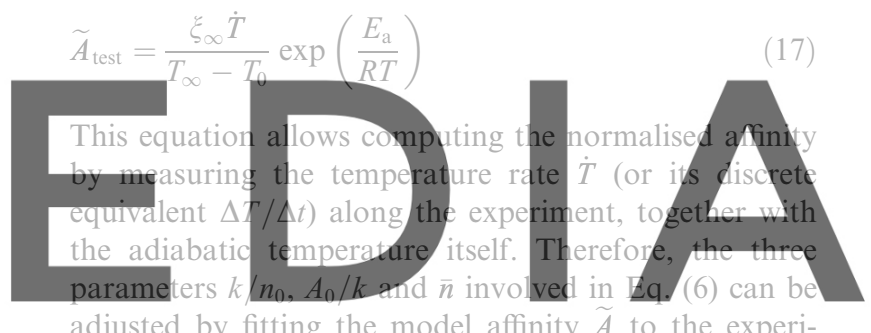

adjusted by fitting the model affinity $A$ to the experidowntoad the version without the watermark

As for the ratio $E_{\mathrm{a}} / R$ it can be experimentally measured as well, ranging within the interval $3000-8000^{\circ} \mathrm{K}$ for concrete. According to $[12,13]$ the activation energy remains almost constant above $20^{\circ} \mathrm{C}$, and consequently we will assume $E_{\mathrm{a}} / R$ as a constant, tuned in order to match the temperature rise under an adiabatic calorimetric test.

\subsection{Application to ordinary and high performance concretes}

Insight on the ability of the proposed model to reproduce the hydration reaction is provided in Fig. 1, where the adiabatic tests conducted by Bentz et al. [14] for an ordinary Portland concrete (OPC) and for a high performance one (HPC) are reproduced. Table 1 reports the values adopted for the various parameters involved in the numerical model. In this figure, as well as in the forthcoming ones, dots correspond to the experimental values, whereas solid lines indicate the predictions from the above described model. From observation of Fig. 1a it becomes evident that for the OPC with a ratio $\mathrm{w} / \mathrm{c}=0.35$ the experimentally measured temperature 

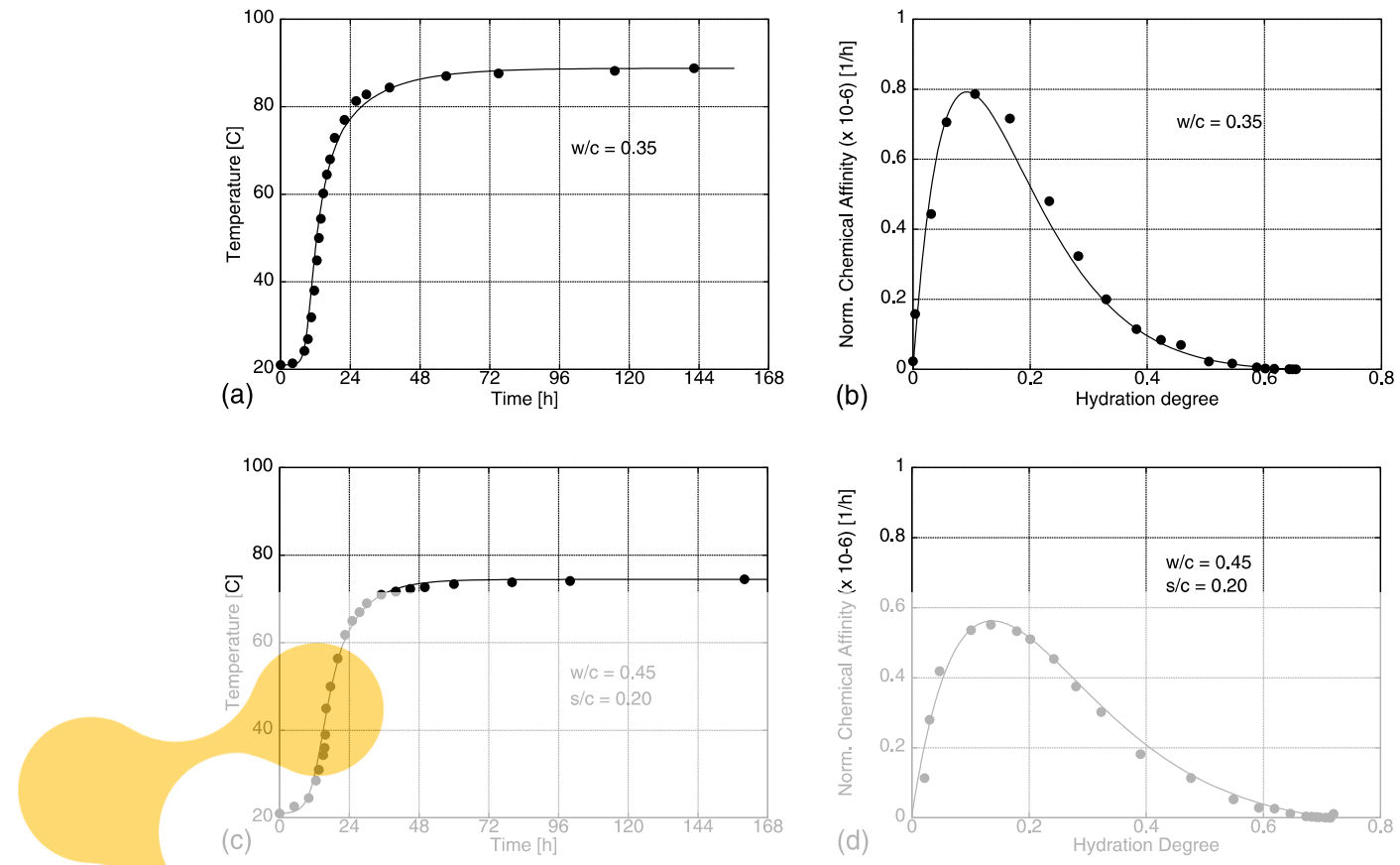

Fig. 1. Temperature evolution and normalised affinity for Bentz
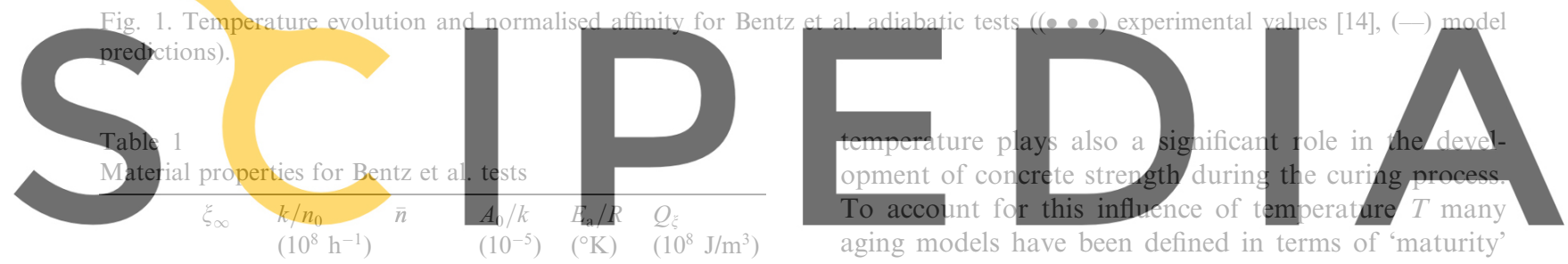

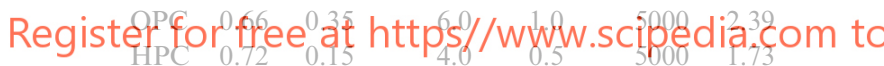
or 'equivalent age' concepts, basically through the defi$\mathrm{C}=2.33 \times 10^{6} \mathrm{~J} / \mathrm{m}^{3}{ }^{\circ} \mathrm{C}, T_{0}=21^{\circ} \mathrm{C}$.

rise is accurately predicted by the numerical model. The same applies for Fig. 1b, where the normalised affinity adopted in the model is compared with the inferred experimental one. Figs. 1c and d provide similar comparisons for the high performance concrete, where $\mathrm{w} / \mathrm{c}=$ 0.45 and a silica fume/cement ratio $\mathrm{s} / \mathrm{c}=0.2$ were considered. Good agreement is therefore obtained for both concretes.

\section{Aging model and temperature effects}

\subsection{Hydration degree and maturity}

From a phenomenological standpoint the development of the mechanical properties of concrete at early ages has to be linked with the hydration degree, as this entity may be viewed as an indicator of how much the inherent chemical reaction has already advanced. However, much experimental data put into evidence that comes superior or inferior to the real one depending on if the actual temperature history has accelerated or retarded the aging process (in comparison with what would occur under a reference temperature $T_{\mathrm{r}}$, typically $20^{\circ} \mathrm{C}$ ). A possible definition for the equivalent age $t_{\mathrm{e}}$ is the one from Freiesleben and Perdersen, revisited in [15],

$t_{\mathrm{e}}=\sum_{i} \exp \left[\frac{E_{\mathrm{a}}}{R}\left(\frac{1}{T_{\mathrm{r}}+273}-\frac{1}{T_{i}+273}\right)\right] \Delta t$

Apart from the fact that the kinetics of the hydration degree is not directly visible on this definition, the assumed accumulative influence of the temperature on the curing process is such that different thermal curing conditions could result in the same maturity, irrespective to the temperature history.

However, as demonstrated in [15] and referred also in $[16,17]$, much experimental observation puts into evidence that concrete strength is affected not only by the accumulative temperature history but also by the history itself. Typically, if a temporary rise on the curing temperature is produced during the first day after casting a 
noticeable acceleration of the hydration process is observed, effect however progressively reduced if the temperature rise is produced at later ages. Conversely, temporary drops on the curing temperature lead to a retarding effect in the hydration reaction, but the influence is also attenuated as the temperature reduction is produced later.

A complementary effect of temperature concerns with the fact that a rise in the early curing temperature improves the early concrete strength, but it reduces its final strength at later ages. On the other hand, through the lowering of the curing temperature at the first ages a retarding of the hydration kinetics is observed, which produces a decay on the initial mechanical properties of concrete, eventually with a subsidiary rise on the limit strength owing to the greater uniformity on the distribution of the hydration products and to the reduced porosity $[16,17]$. These reasoning put into evidence the limited 'memory' proficiency of traditional formulae like the one reproduced in Eq. (18), since concretes cured under different temperature histories could have the same maturity and consequently the same final strength, an assumption in contradiction with the experimental evidence. Therefore a realistic aging model should ac-

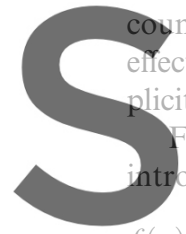
nt for the influence of the of the curing temperature icitly included as well.

Focusing in the concrete duce an internal variable

$f(\kappa)=\kappa f_{\infty}$

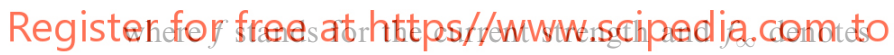
the final compressive strength at the reference temperature. As defined here variable $k$ corresponds to a normalised strength, hereinafter referred to as an aging degree. Since we intend to account for both the hydration degree and the temperature effects on the evolution of the compressive strength of concrete, let us postulate a rate equation for the aging degree of the form

$\dot{\kappa}=\lambda_{T}(T) \lambda_{\xi}(\xi) \dot{\xi}$ where $\lambda_{T} \lambda_{\xi} \geqslant 0$. Factor $\lambda_{\xi}$ is supposed here to obey to a linear expression of the form

$\lambda_{\xi}=A \xi+B$

where $A$ and $B$ are material parameters. Factor $\lambda_{T}$ accounts for the influence of temperature, here reproduced by the expression

$\lambda_{T}=\left(\frac{100-T}{100-T_{\mathrm{r}}}\right)^{a}$

where $T_{\mathrm{r}}$ is the reference temperature for the determination of $f_{\infty}$ (typically $20^{\circ} \mathrm{C}$ ) and the exponent $a$ is a model parameter that serves to regulate the thermal incidence on the evolution of the aging degree. Note that for $T>T_{\mathrm{r}}$ term $\lambda_{T}$ becomes smaller than one, thus attenuating the strength increase; conversely for $T<T_{\mathrm{r}}$ it results $\lambda_{T}>1$, thus enhancing the evolution of the aging degree. Fig. 2 illustrates the typical evolutions obtained for $\xi$ and $f$ when curing takes place under three different isothermal conditions: 5, 20 and $40{ }^{\circ} \mathrm{C}$. Note in Fig. 2a that the final value for the hydration degree is the same for the three curing temperatures, and consequently the maturity and equivalent age models can only reproduce

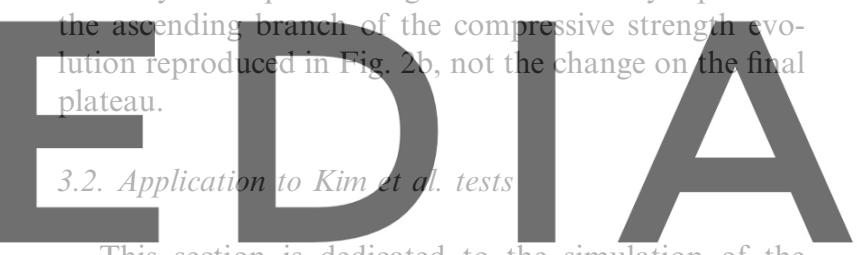

This section is dedicated to the simulation of the experimental tests reported by Kim et al. in [15], which dowerd pad the version withoutacthe twatermark on the evolution of the compressive strength of concrete at early ages, as well as on its final values. The curing was carried out under controlled isothermal conditions during the first 28 days, namely at the temperatures of 5 , 20 and $40{ }^{\circ} \mathrm{C}$, as depicted on the first three shadowed rows of Fig. 3. Furthermore, a complex experimental program was pursued around the curing temperature of $20{ }^{\circ} \mathrm{C}$, consisting in raising it up to $40{ }^{\circ} \mathrm{C}$ or lowering it until $5{ }^{\circ} \mathrm{C}$ during just one day, and returning to the
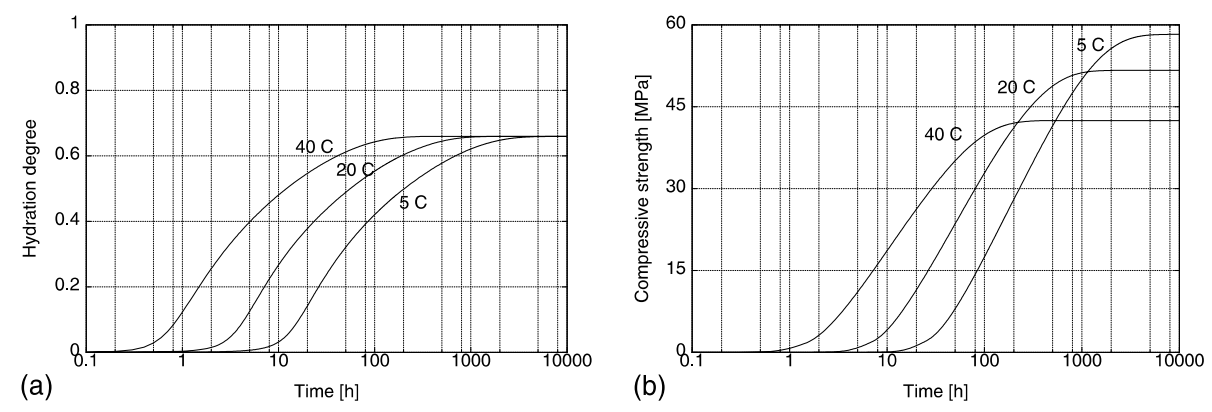

Fig. 2. Influence of temperature on the hydration and aging phenomena. 


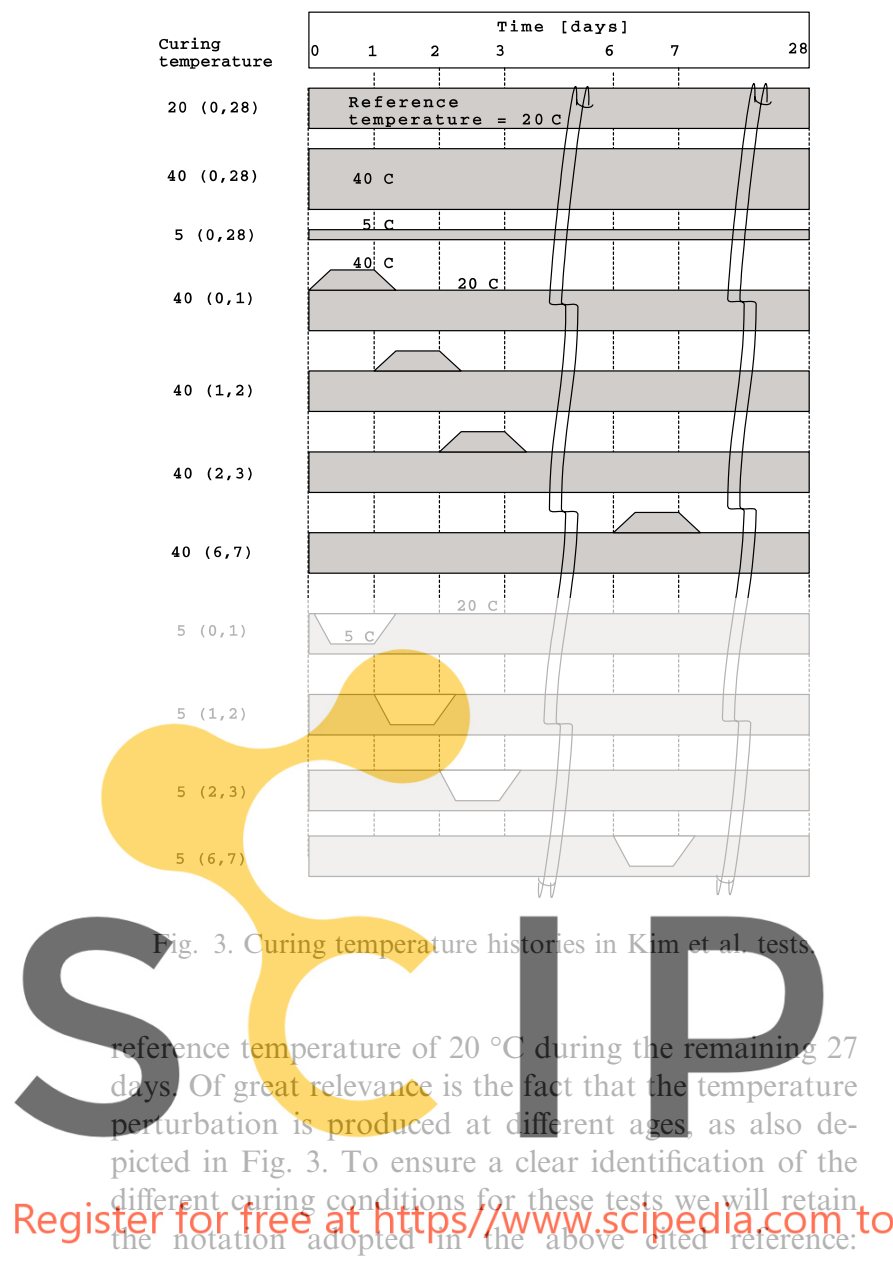

$40(d, d+1)$ point out that a raise from 20 to $40{ }^{\circ} \mathrm{C}$ is produced between days $d$ and $d+1$, whereas $5(d, d+1)$ indicate a decay from 20 to $5{ }^{\circ} \mathrm{C}$ during the same period (see also Fig. 3 for clarification). It is here emphasised that in spite of the temporary variations introduced in the curing temperature these tests can be considered as 'stepwise' isothermal. Two different w/c ratios of 0.35 and 0.55 were considered for the above referred tests, reproduced by the numerical model with the material properties described in Table 2.

Focusing on the first three curing conditions of Fig. 3, Figs. $4 \mathrm{a}$ and $\mathrm{b}$ allow to compare the compressive strength evolutions predicted by the numerical model against the ones obtained during the experimental tests, for the two different $\mathrm{w} / \mathrm{c}$ ratios. The overall agreement of the aging model predictions with the test results is fairly good, since it captures the double influence of the curing temperature: (i) as an activator of the hydration reaction and (ii) as a conditioning factor of the final concrete strength.

The next figures concern the remaining tests sketched in Fig. 3: Fig. 5 is dedicated to the $40(0,1), 40(1,2)$, $40(2,3)$ and $40(6,7)$ tests, whereas Fig. 6 reproduces the $5(0,1), 5(1,2), 5(2,3)$ and $5(6,7)$ ones, all of them plot-

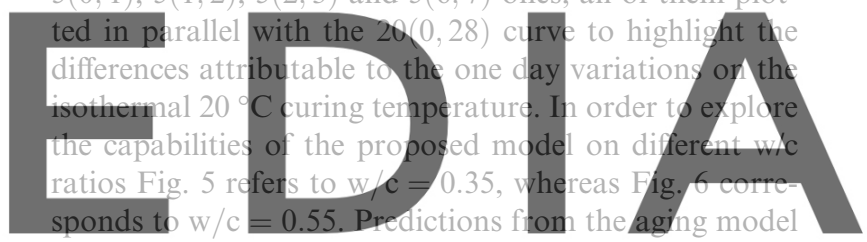
compare remarkably well with the experimental obser-

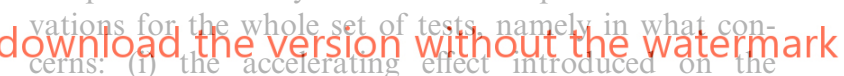

Table 2

Material properties for Kim et al. tests

\begin{tabular}{llllllllllll}
\hline $\mathrm{w} / \mathrm{c}$ & $\xi_{\infty}$ & $k / n_{0}\left(10^{10} \mathrm{~h}^{-1}\right)$ & $\bar{n}$ & $A_{0} / k\left(10^{-5}\right)$ & $E_{\mathrm{a}} / R\left({ }^{\circ} \mathrm{K}\right)$ & $Q_{\xi}\left(10^{8} \mathrm{~J} / \mathrm{m}^{3}\right)$ & $A$ & $B$ & $\xi_{\text {set }}$ & $f_{\infty}(\mathrm{MPa})$ & $a$ \\
\hline 0.35 & 0.66 & 4.33 & 5.8 & 1.0 & 7000 & 1.25 & 3.79 & -1.17 & 0.2 & 52 & 0.7 \\
0.55 & 0.76 & 1.20 & 7.5 & 0.1 & 6500 & 1.25 & 3.72 & -1.85 & 0.3 & 42 & 0.9 \\
\hline
\end{tabular}

$C=2.07 \times 10^{6} \mathrm{~J} / \mathrm{m}^{3}{ }^{\circ} \mathrm{C}, T_{0}=21{ }^{\circ} \mathrm{C}, T_{\mathrm{r}}=20^{\circ} \mathrm{C}$.
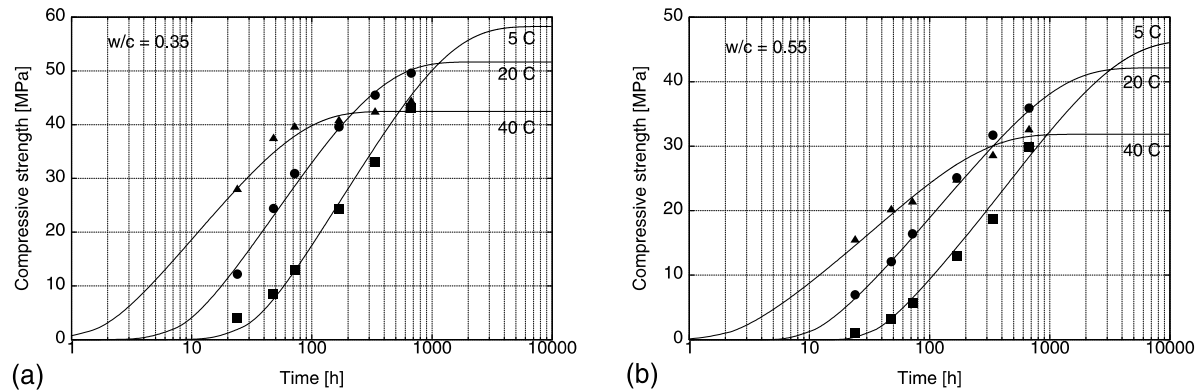

Fig. 4. Strength evolution for the isothermal Kim et al. tests. 

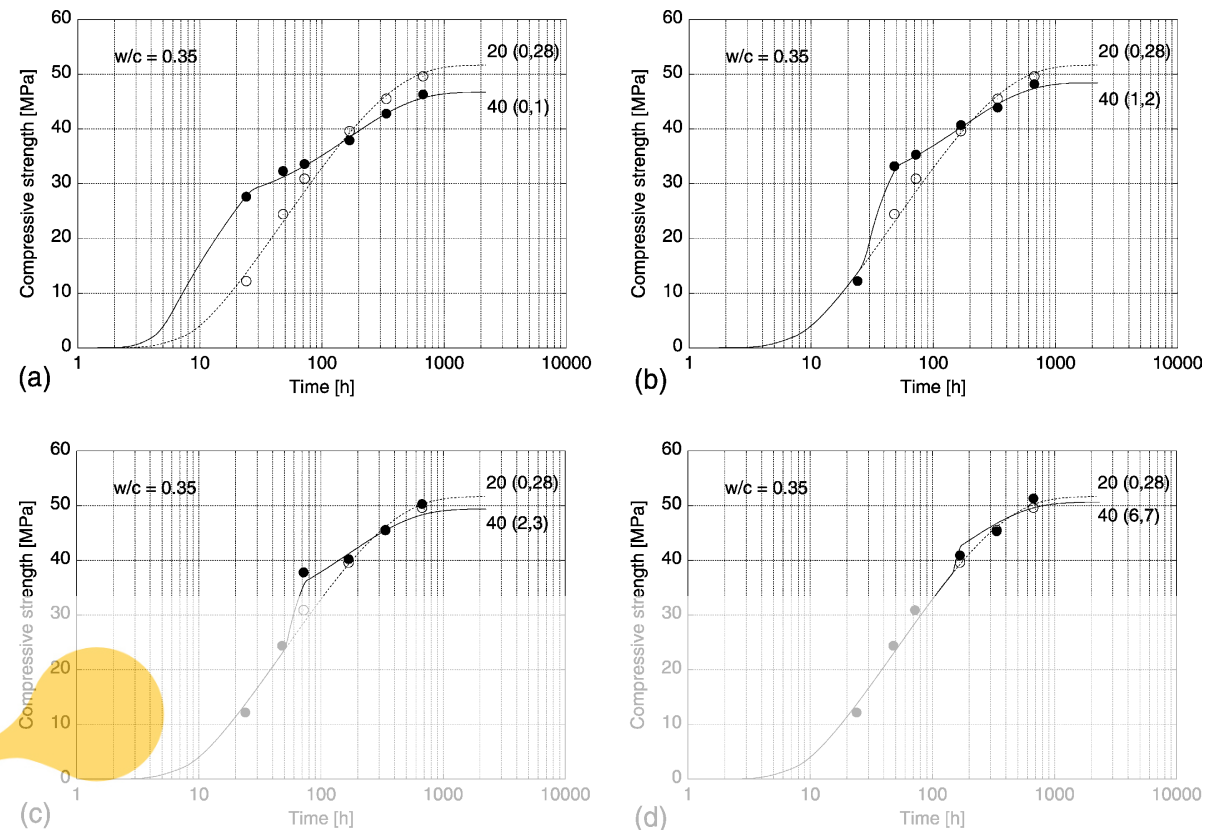

Fig. 5. Strength evolution for the $\mathrm{w} / \mathrm{c}=0.35 \mathrm{Kim}$ et al. tests.
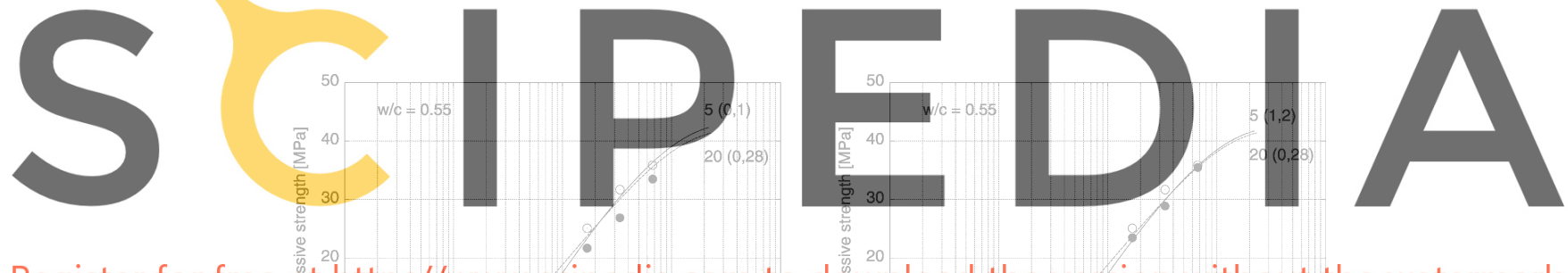

Register for free a

(a)

$\stackrel{0}{1}$

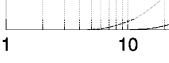

100
Time $[\mathrm{h}]$

$1000 \quad 10000$

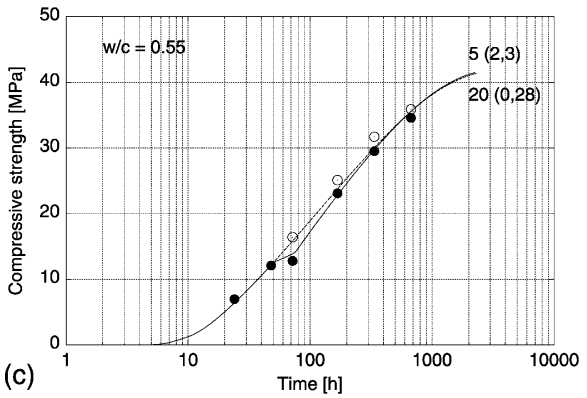

(b)
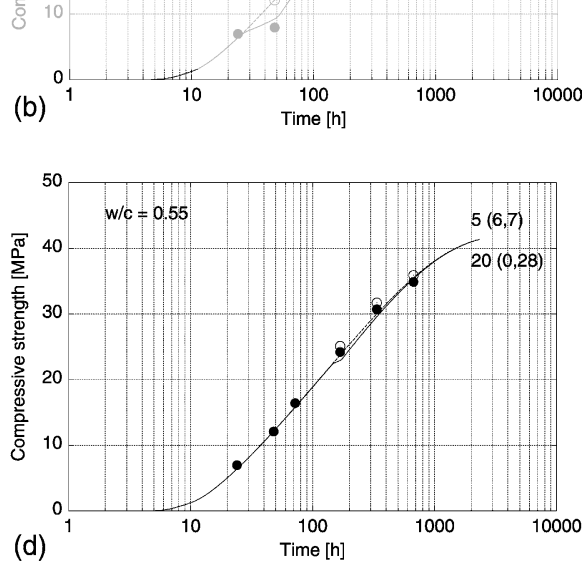

Fig. 6. Strength evolution for the $\mathrm{w} / \mathrm{c}=0.55 \mathrm{Kim}$ et al. tests.

compressive strength evolution due to the elevation of the curing temperature above the $20^{\circ} \mathrm{C}$, or conversely, the retarding effect produced on concrete aging due to reducing the temperature to $5{ }^{\circ} \mathrm{C}$, (ii) the progressive attenuation of the influence of the one day thermal variations as such variations are produced on later ages, practically cancelling after the 6-7th day, irrespective to the w/c ratio and the stepwise thermal change. 


\section{Application to a concrete deck}

Fig. 7 illustrates the configuration of the viaducts of the Öresund Link, between Denmark and Sweden. The viaduct is made up by two steel truss beams, on top of which a concrete deck is connected. By virtue of the extreme environmental conditions expected for the site of the Öresund Link - temperatures below $-20^{\circ} \mathrm{C}$, marine atmosphere, cycles of freeze-thaw-quite demanding casting and curing conditions were specified for the decks, which compelled the constructor (Dragados offshore) to build a curing warehouse for protecting the viaducts, inside of which the air temperature was kept around $20^{\circ} \mathrm{C}$ via an acclimatization system. In order to control the casting and the curing conditions several prototynes of the viaducts were monitored via thermal sensors, whose temperature measurements will be used to validate the described numerical model.

Fig. 8 depicts the left half of the viaduct concrete deck, as well as the four-noded FE mesh adopted for the discretization and the location of the four thermal sen- sors (sensors 1, 6, 10 and 11) adopted for comparison of the experimental measurements with the numerical predictions. Computations started with the calibration of the hydration and aging model parameters, task that was undertaken on the basis of the concrete characterization performed in the laboratory that supported the constructor, and lead to the set of model parameters resumed in Table 3. The performed calibration is reproduced in Fig. 9a, which compares the 'numerical' and 'experimental' temperature rises for a concrete sample cured in adiabatic conditions, and in Fig. 9b, which depicts the numerical and experimental evolutions foreseen for the compressive strength during a $20{ }^{\circ} \mathrm{C}$ isothermal test.

The thermal analysis performed for simulating the curing of the concrete deck is reported in Fig. 10, where the temperature evolutions foreseen by the numeric model are compared with the experimentally measured ones at sensors 1, 6, 10 and 11. For this numerical simulation two thermal contact conditions were considered along the deck boundaries: (i) one at the top,
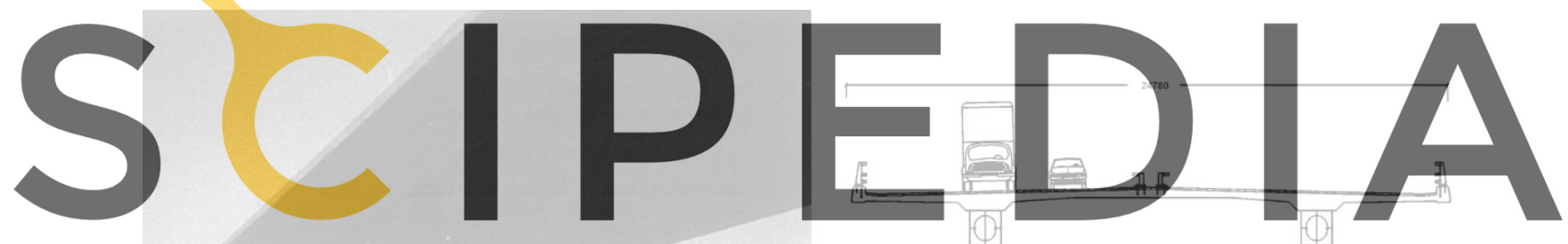

Register for free at https//www.scipedia.com to
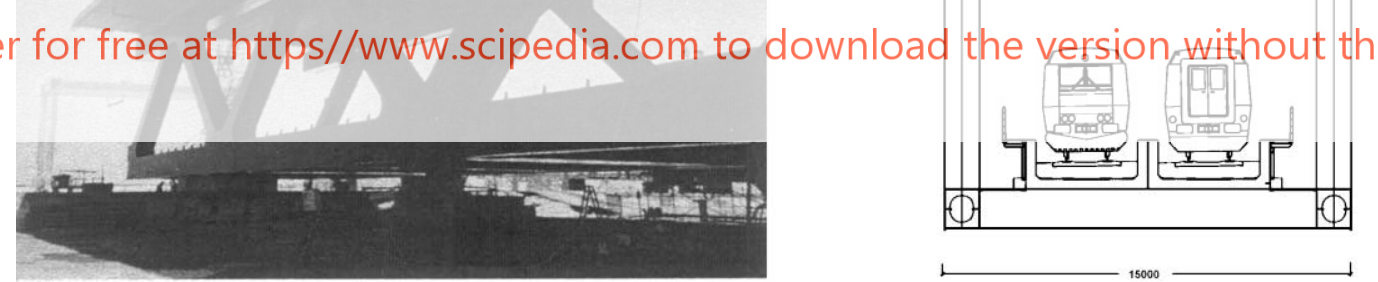

Fig. 7. Öresund Link viaduct.

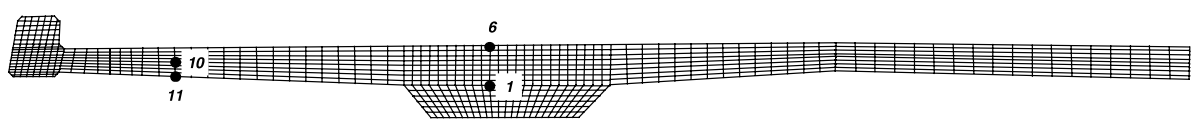

Fig. 8. Four-noded FE mesh and location of the thermal sensors.

Table 3

Material properties for the Öresund concrete deck

\begin{tabular}{|c|c|c|c|c|c|c|c|c|c|c|c|}
\hline $\mathrm{w} / \mathrm{c}$ & $\xi_{\infty}$ & $k / n_{0}\left(10^{7} \mathrm{~h}^{-1}\right)$ & $\bar{n}$ & $A_{0} / k\left(10^{-4}\right)$ & $E_{\mathrm{a}} / R\left({ }^{\circ} \mathrm{K}\right)$ & $Q_{\xi}\left(10^{8} \mathrm{~J} / \mathrm{m}^{3}\right)$ & $A$ & $B$ & $\xi_{\text {set }}$ & $f_{\infty}(\mathrm{MPa})$ & $a$ \\
\hline 0.32 & 0.64 & 0.27 & 8.4 & 1.0 & 4000 & 1.95 & 2.19 & 0.16 & 0.1 & 53.4 & 0.0 \\
\hline
\end{tabular}

$C=1.87 \times 10^{6} \mathrm{~J} / \mathrm{m}^{3}{ }^{\circ} \mathrm{C}, T_{0}=20^{\circ} \mathrm{C}, T_{\mathrm{r}}=20^{\circ} \mathrm{C}$. 

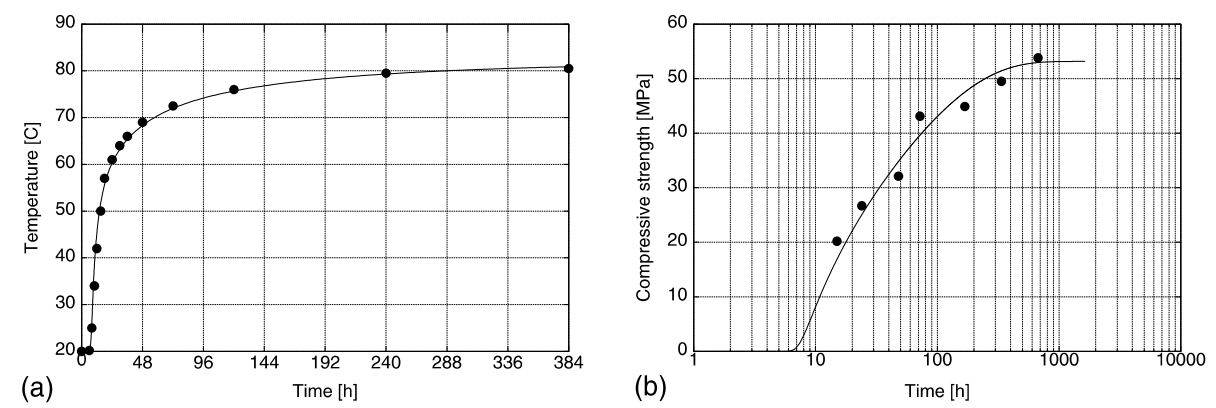

Fig. 9. Calibration tests: (a) temperatures on an adiabatic test; (b) compressive strength evolution on a $20^{\circ} \mathrm{C}$ isothermal test.
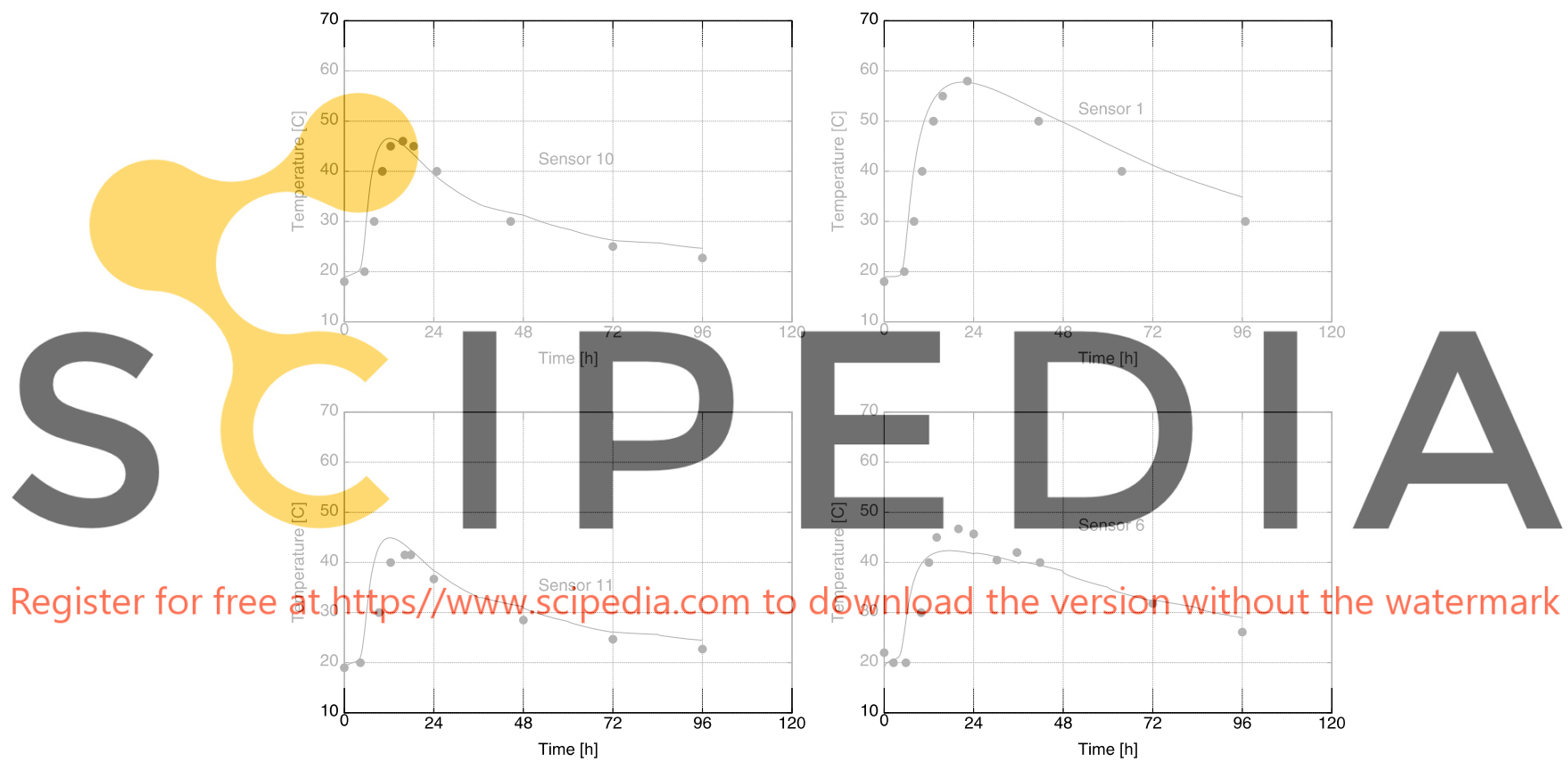

Fig. 10. Evolution of temperature during the curing of the concrete deck.

corresponding to the plastic sheet which recovered the concrete surface during the casting and curing process, and (ii) one at the bottom, representing the formwork conductivity properties (for further details see [11]).

As demonstrated in Fig. 10 the numeric model provides an adequate prediction of the overall concrete curing phenomenon, expressed through the reasonably accurate guessing of the temperature evolutions registered at the four sensors. It is remarked the fast temperature rise during the first day after casting, with a maximum temperature jump of about $40{ }^{\circ} \mathrm{C}$ at sensor 1 , which is closer to adiabatic conditions by virtue of the largest deck thickness and of the internal placing of the sensor. Fig. 10 demonstrates that the proposed model is also able to predict the cooling phase which follows the initial heating process, a relevant feature since associated to the contraction of the concrete deck, therefore associated to the undesirable generation of tensile stresses. Note that the heating and the cooling phases perform differently from one sensor to the other, yet reasonably captured by the numerical model, putting into evidence the ability of the latter in providing physically sound predictions of both the time and the spatial temperature distributions induced by the concrete curing.

\section{Conclusions}

With a format suitable for structural applications a model that accounts for the hydration phenomenon at a macroscopic level is proposed in this work. It calls for a normalised variable termed hydration degree, for which 
an evolution law is presented. In order to cope with structural applications the thermal problem arising from the internal heat source provided by the hydration reaction needs to be solved, which is accomplished by adopting a FE spatial discretization and a time integration based on the explicit Euler scheme. Through comparison with suitable experimental tests performed for ordinary and high performance concretes accurate predictions for the hydration degree and the temperature evolutions are obtained during the curing process.

As for the evolution of the concrete compressive strength the novel concept of aging degree is introduced, accounting for both the hydration degree and the temperature influences on the aging of concrete during the curing. An important feature easily captured by the proposed aging model is the temperature influence on the final concrete strength, that is, at the end of the hydration reaction. Model capabilities are checked by comparing several experimentally observed strength evolutions at the early ages of concrete with the ones obtained numerically.

Suitability of the proposed numerical model for real structural applications is demonstrated by simulating the concrete curing reported for an experimentally instrumented viaduct deck. This application puts into evidence the ability of the proposed model to provide accurate predictions of the temperature evolutions registered on several thermal sensors installed on the deck.

\section{Acknowledgements}

This research was partially supported by the Training and Mobility of Researchers Programme, Access to Large Installations, under contract ERBFMGECT950062 "Access to supercomputing facilities for european researchers" established between the European Community and CESCA-CEPBA.

\section{References}

[1] Coussy O. Mechanics of porous media. Chichester: John Wiley \& Sons; 1995.

[2] Ulm FJ, Coussy O. Modelling of thermo-chemo-mechanical couplings of concrete at early ages. ASCE J Eng Mech 1995;121(7):785-94.
[3] Ulm FJ, Coussy O. Strength growth as chemo-plastic hardening in early age concrete. ASCE J Eng Mech 1996;122(12):1123-32.

[4] Pantazopoulo SJ, Mills RH. Microstructural aspects of the mechanical response of plain concrete. ACI Mater J 1995;92(6):605-16.

[5] Acker P. Comportement mécanique du béton: apports de l'approche physico-chimique. In: Rapport de recherche N. 152. Paris: Laboratoire Ponts et Chaussées, 1988.

[6] de Schutter G, Taerwe L. General hydration model for Portland cement and blast furnace slag cement. Cement Concrete Res 1995;25(3):593-604.

[7] Cervera M, Oliver J, Prato T. A thermo-chemo-mechanical model for concrete. I: Hydration and aging. ASCE J Eng Mech 1999;125(9):1018-27.

[8] Reinhardt HW, Blaauwendraad J, Jongedijk J. Temperature development in concrete structures taking account of state dependent properties. In: Proceedings of the International Conference of Concrete at Early Ages. 1982.

[9] Rostassy FS, Gustsch A, Laube M. Creep and relaxation of concrete at early ages. Experiments and mathematical modelling. In: Mang H, Bicanic N, de Borst R, editors. Proceedings of the 5th International RILEM Symposium on Creep and Shrinkage of Concrete. EFN Spon; 1993. p. $453-8$.

[10] Torrenti JM, Ghenot I, Laplante P, Acker P, Larrand F. Numerical simulation of temperatures and stresses in concrete at early ages. In: Bazant ZP, Carol I, editors. Proceedings of the International Conference on Computational Modelling of Concrete Structures. Swansea: Pineridge Press; 1994. p. 559-68.

[11] Prato T. Concrete behaviour during the early ages. Modelling and applications. PhD Thesis, ETSECCPB, UPC, Barcelona, 1999 [in Spanish].

[12] Hansen PF, Nielsen A. Method for quick calculation of temperature differences in concrete members. VTT Symposium 61, Espoo, 1985.

[13] van Breugel K. Hysmostruc: a computer based simulation model for hydration and formation of structure in cement based materials. In: Nonat R, Mutin JC, editors. Hydration and setting of cements. London: RILEM; 1992. p. 361-8.

[14] Bentz DP, Waller V, de Larrard F. Prediction of adiabatic temperature rise in conventional and high-performance concretes using a 3D microstructural model. Cement Concrete Res 1998;28(2):285-97.

[15] Kim J-K, Moon Y-H, Eo S-H. Compressive strength development of concrete with different curing time and temperature. Cement Concrete Res 1998;28(12):1761-73.

[16] Kjellsen KO, Detwiler RJ. Later-age strength prediction by a modified maturity model. ACI Mater J 1993;90(3):220-7.

[17] Carino NJ. Temperature effects on the strength-maturity relation of mortar. In: Report NBSSIR 81-2244. Washington DC: National Bureau of Standards; 1981. 